\title{
Ferulic Acid, A Potential Antithrombotic Drug
}

\author{
Shao Shuai ${ }^{1}, 2$, Gao Yue ${ }^{2 *}$ \\ 'National Institute for Radiological Protection, Chinese Center for Disease Control and Prevention, Beijing 100088, China \\ ${ }^{2}$ Beijing Institute of Radiation Medicine, Beijing 100850, China
}

Article Info

\section{Article Notes}

Received: April 04, 2018

Accepted: May 10, 2018

\section{${ }^{*}$ Correspondence:}

Prof. Gao Yue, Beijing Institute of Radiation Medicine, Tai-Ping

Road, Beijing 100850, China;

Telephone: +86-10-66931312;

Email: gaoyue@bmi.ac.cn

(C) 2018 Yue G. This article is distributed under the terms of the Creative Commons Attribution 4.0 International License.

\section{Keywords}

Ferulic Acid

Antithrombotic drug

Coagulation

Platelet Aggregation

Adhesion Factor

Vascular Endothelial Cell

\section{ABSTRACT}

Coagulation abnormalities are critical diseases that threaten the survival of patients. Ferulic acid can regulate the blood coagulation function in two aspects. This paper reviews and discusses the reported mechanisms of the antithrombotic activities of ferulic acid. Previous studies suggested that ferulic acid played a role in antithrombosis by inhibiting platelet aggregation and protecting the endotheliocyte. Meanwhile, ferulic acid has fewer side effects on the platelet, leukocytosis and gastrointestinal tract, because it can promote the formation and differentiation of hematopoietic progenitor cells and protect the intestinal cells from injury. Therefore, ferulic acid is a potential protector from thrombotic diseases, such as cardiovascular dysfunction, pulmonary thromboembolism and deep vein thrombosis.

\section{Introduction}

Thrombosis, which is an abnormal intravascular coagulation, is a critical threat to the survival of patients with infection, trauma, hypothermia, hyperpyrexia and radiation sickness ${ }^{1,2}$. This abnormality results in cardiovascular diseases (coronary artery disease, stroke, peripheral artery disease, thromboembolic disease and venous thrombosis), pulmonary embolism, deep vein thrombosis and disseminated intravascular coagulation. Several anticoagulant drugs, such as aspirin and clopidogrel, are widely used in the clinic. However, the negative side effects due to longterm administration of these drugs, including thrombocytopenia, leukopenia, bleeding and gastrointestinal reaction, cannot be neglected ${ }^{3}$. Thus, anticoagulant drugs without these side effects are urgently needed clinically.

Ferulic acid (FA) is an important polyphenol in many traditional Chinese medicines, such as Chuanxiong Rhizoma and Angelica Radix. This compound can accelerate blood circulation and remove stasis. FA has recently attracted increased attention because of its curative effect on cardiovascular diseases. FA plays an important role not only in the inhibition of platelet aggregation ${ }^{4,5}$ but also in the boosting of platelet count ${ }^{6}$ and protection of vascular endothelial cells ${ }^{7,8}$ (Fig. 1). Therefore, FA could be further developed as a new agent with high efficacy and fewer side effects to mitigate thrombosis.

This paper presents a review of the medical literature of the effects of FA, including the inhibition of platelet aggregation and thrombocytopenia and the protection of vascular endothelial cells. The results were used to analyze the antithrombotic mechanism of FA. In addition, we assessed the side effects of this compound. 


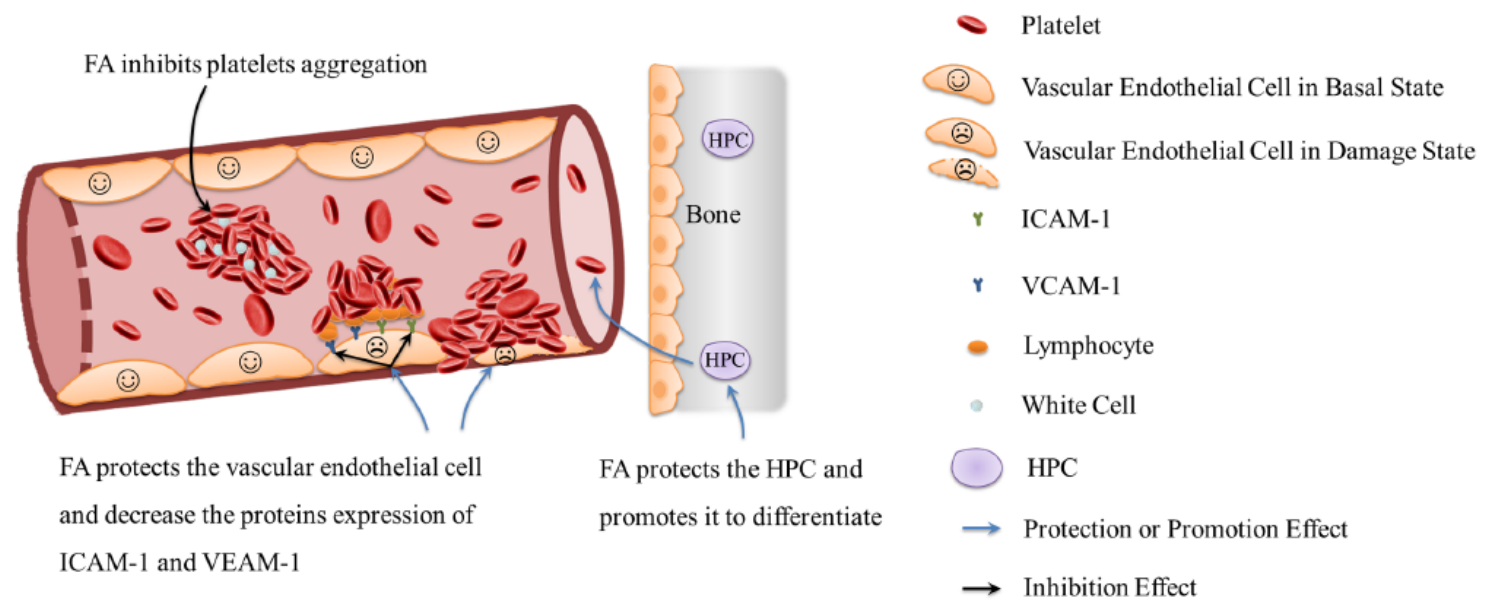

Figure 1. Effect of ferulic acid (FA) on thrombosis

FA can be an antithrombotic drug via anti-aggregation, endotheliocyte-protection and down-regulation of adhesion factors (ICAM-I,

VEAM-I, and so on). Moreover, FA has fewer side effects. For example, in thrombocytopenia, FA protects the hematopoietic progenitor cell (HPC) and promotes it to differentiate into the platelet.

\section{Inhibition effect of ferulic acid on platelet aggregation}

Platelets are principally involved in normal hemostasis, pathological bleeding and thrombosis ${ }^{9}$. Platelet aggregation causes thrombotic diseases, such as pulmonary embolism and atherosclerosis ${ }^{10,11}$. According to statistics, antiplatelet aggregation drugs cover $64.49 \%$ market share ${ }^{12}$. An inhibitor of platelet aggregation, such as aspirin, is the first-choice medicine and the mainstream first-line drug to prevent and cure thrombus diseases ${ }^{13}$. Similar to aspirin, FA significantly decreased the mortality from pulmonary thrombosis in mice ${ }^{4,14}$. Meanwhile, FA also prolonged the tail bleeding and clotting time in mice. FA slightly altered the activated partial thromboplastin time, prothrombin time and thrombin time in rats. These coagulation parameters and platelets are the most important two factors in intravascular coagulation. The changes in the coagulation parameters suggest that the antithrombotic activities of FA may be regulated by inhibiting platelet aggregation rather than by inhibiting the release of thromboplastin or formation of thrombin.

Hong et al. showed that FA inhibited platelet aggregation via intracellular cyclic nucleotide signaling ${ }^{4}$. Platelet aggregation and ATP release induced by various agonists, such as adenosine diphosphate (ADP), thrombin, U46619, and arachidonic acid, were dose-dependently inhibited by FA $(50-200 \mu \mathrm{M})$ both in vivo and in vitro with statistical significance. For the platelet induced by agonists, FA attenuated the intracellular calcium ion $\left(\mathrm{Ca}^{2+}\right)$ mobilization and elevated the cyclic adenosine monophosphate (cAMP) expression. The decrease in thromboxane A2 $\left(\mathrm{TXA}_{2}\right)$ was related to calcium inhibition and cAMP elevation. Thus, the changes in these molecules further induced the reduction in thromboxane $B_{2}\left(\mathrm{TXB}_{2}\right)$, which is a stable metabolite of $\mathrm{TXA}_{2}$. On the other hand, FA upregulated the expression of cAMP, which results in the decrease in platelet activation and aggregation mediated by an agonist. Vasodilator-simulated phosphoprotein is a substrate of the cyclic nucleotide, and phosphodiesterase (PDE) is an enzyme family responsible for the hydrolysis of cAMP/ cyclic guanosine monophosphate (cGMP). FA upregulated the expression of cAMP, cGMP, and phosphorylated VASP but downregulated phosphor-mitogen-activated protein kinase and PDE in washed rat platelets. Furthermore, FA attenuated platelet agonists by inhibiting the P38 and ERK2 phosphorylation and activating PKA and PKG via inhibition of PDE.

\section{Protection effect of ferulic acid on vascular endothelial cells}

The vascular endothelial cell is a critical part of the vessel wall, especially in the alternative translations. This cell resides at the interface between blood and surrounding tissues ${ }^{15}$. Thus, the vascular endothelial cell serves an essential role in thrombosis formation. An injured vascular endothelial cell expresses an excess of adhesion factors. These factors promote the adhesion of leukocyte to the endotheliocyte and platelets ${ }^{16}$. The complexes of leukocyte and endotheliocyte /platelet regulate the activation of platelets and the coagulation cascade ${ }^{17}$. Therefore, the excessive activation of leukocytes during the stress response and the adhesion of the activated leukocyte with the endotheliocyte/platelet play an important role in the activation of the coagulation cascade. These two processes may affect the blood flow and thrombus formation and induce a systemic procoagulant state. In capillary 
circulation, the initial adhesion of activated platelets to the endothelium is mediated by von Willebrand factor (vWF) and ADPase. FA attenuates the expression of intercellular cell adhesion molecule 1, vascular cell adhesion molecule 1 and vWF via the JNK signaling pathway. Moreover, FA inhibits radiation-induced lymphoma adhesion to the endotheliocyte. Ma et al. demonstrated these phenomena using radiation-induced $\mathrm{HUVEC}^{8}$. In addition, they found that FA increased the cell viability of HUVECs ${ }^{7}$. Adhesion factors and collagen fibers are the two key factors of thrombosis in vascular endothelial cells. The collagen fibers are exposed after a more serious damage to the vascular endothelial cell ${ }^{18}$. Platelets adherent to the phosphatidylserine are expressed by the exposed collagen fibers. In intimate contact with collagen fibers, activated platelets are accreted in layers and have been recruited and activated by secreted products, including $\mathrm{TXA}_{2}, \mathrm{TXB}_{2}$, and $\mathrm{ADP}^{19}$. Hong et al. suggested that FA dose-dependently decreased $\mathrm{TXB}_{2}$ production activated by collagen or $\mathrm{ADP}^{4}$. Thus, vascular endothelial cell protection is another important evidence of the antithrombotic activity of FA.

\section{Side effects of ferulic acid on platelets, leukocytosis, and gastrointestinal}

Thrombocytopenia, aleucocytosis, bleeding, and gastrointestinal reaction are the main side effects of clinically available antithrombotic drugs. In contrast, FA has bidirectional regulation effects on platelet and leukocyte. Ma et al. found that FA significantly increased the recovery of platelets and leukocytosis because it increased the formation of clones of hematopoietic progenitor cells ${ }^{6}$. These hematopoietic cells can differentiate into platelet and leukocyte under the induction by the corresponding cytokines $^{20-22}$. Moreover, in the concentration range of 0-500 nM, FA had no cytotoxic effect on IEC-6 but exhibited a protective effect against heat stress-induced intestinal epithelial barrier dysfunction ${ }^{23}$. Consequently, FA is expected to become an antithrombotic drug with fewer side effects.

\section{Summary}

Thrombotic diseases can be divided into arterial, venous and capillary thromboses. FA is a candidate for the treatment of thrombotic diseases. As a potential antithrombotic drug, FA not only inhibits platelet aggregation but also protects the vascular endothelial cells. Moreover, compared with aspirin, FA shows no side effects on the platelet, leukocytosis and gastrointestinal tract. Therefore, FA could be further developed as a drug against thrombosis.

\section{Funding information and conflict of interest}

This work was supported by the National Natural Science Foundation of China (No.81130067 and No.81303316).
The authors have no conflicts of interest that are directly relevant of the content of this manuscript.

\section{References}

1. Dalton HJ, Garciafilion P, Holubkov R, et al. Association of bleeding and thrombosis with outcome in extracorporeal life support. Pediatric critical care medicine: a journal of the Society of Critical Care Medicine and the World Federation of Pediatric Intensive and Critical Care Societies. 2015; 16: 167-174.

2. Fiona A. Stewart JAMT. Radiation Nephropathy\&The Link Between Functional Damage and Vascular Mediated Inflammatory and Thrombotic Changes. ACTA ONCOL. 2001; 40: 952-957.

3. Robertson L. Antiplatelet and anticoagulant drugs for prevention of restenosis/reocclusion following peripheral endovascular treatment. Cochrane Database Syst Rev. 2012; 8: D2071.

4. Hong $\mathrm{Q}$ Ma ZC, Huang $\mathrm{H}$, et al. Antithrombotic activities of ferulic acid via intracellular cyclic nucleotide signaling. EUR J PHARMACOL. 2016; 777: 1-8.

5. Choi J, Park J, Kim K, et al. In vitro and in vivo antithrombotic and cytotoxicity effects of ferulic acid. J BIOCHEM MOL TOXIC. 2018; 32 : e22004.

6. Ma ZC, Hong Q, Wang YG, et al. Effects of ferulic acid on hematopoietic cell recovery in whole-body gamma irradiated mice. INT J RADIAT BIOL. 2011; 87: 499-505.

7. Ma ZC, Hong Q Wang YG, et al. Ferulic acid protects human umbilical vein endothelial cells from radiation induced oxidative stress by phosphatidylinositol 3-kinase and extracellular signal-regulated kinase pathways. BIOL PHARM BULL. 2010; 33: 29-34.

8. Ma ZC, Hong Q, Wang YG, et al. Ferulic acid attenuates adhesion molecule expression in gamma-radiated human umbilical vascular endothelial cells. BIOL PHARM BULL. 2010; 33: 752-758.

9. Becker RC. Platelet Biology: The Role of Platelets in Hemostasis, Thrombosis and Inflammation. AM J CARDIOL. 2014; 83: 3E-6E.

10. Sevuk U, Bahadir MV, Altindag $R$, et al. Value of serial platelet indices measurements for the prediction of pulmonary embolism in patients with deep venous thrombosis. Therapeutics \& Clinical Risk Management. 2015; 11: 1243.

11. Ryszawa N, Kawczyńskadrózdz A, Pryjma J, et al. Effects of novel plant antioxidants on platelet superoxide production and aggregation in atherosclerosis. Journal of Physiology \& Pharmacology An Official Journal of the Polish Physiological Society. 2006; 57: 611.

12. Steinhubl SR, Berger PB, Rd MJ, et al. Early and sustained dual oral antiplatelet therapy following percutaneous coronary intervention: a randomized controlled trial. Acc Current Journal Review 2002; 12: 78-79.

13. Manolis AS, Tzeis S, Andrikopoulos G, et al. Aspirin and clopidogrel: a sweeping combination in cardiology. Current Medicinal Chemistry Cardiovascular \& Hematological Agents 2005;3:-.

14. Zhang PX, Lin H, Qu C, et al. Design, Synthesis, and In Vitro Antiplatelet Aggregation Activities of Ferulic Acid Derivatives. J CHEM-NY. 2015; 2015: 1-7.

15. Yau JW, Teoh H, Verma S. Endothelial cell control of thrombosis. BMC CARDIOVASC DISOR. 2015; 15: 130.

16. Afshar-Kharghan V, Thiagarajan P. Leukocyte adhesion and thrombosis. CURR OPIN HEMATOL. 2006; 13: 34.

17. Stefanini L, Bergmeier W. Negative regulators of platelet activation and adhesion. Journal of Thrombosis \& Haemostasis. 2017.

18. Greineder CF, Johnston IH, Villa $\mathrm{CH}$, et al. ICAM-1-targeted thrombomodulin mitigates tissue factor-driven inflammatory thrombosis in a human endothelialized microfluidic model. Blood Advances. 2017; 1: 1452. 
19. Farndale RW, Sixma JJ, Barnes MJ, et al. The role of collagen in thrombosis and hemostasis. J THROMB HAEMOST. 2004; 2: 561-573.

20. Pietrzyknivau A, Poiraultchassac S, Gandrille S, et al. ThreeDimensional Environment Sustains Hematopoietic Stem Cell Differentiation into Platelet-Producing Megakaryocytes. PLOS ONE. 2015; 10: e136652.

21. Sioud M, Fløisand Y. NOD2/CARD15 on bone marrow CD34+ hematopoietic cells mediates induction of cytokines and cell differentiation. J LEUKOCYTE BIOL. 2009; 85: 939.
22. Bal G, Fabian D, Maia D, et al. Effect of thrombopoietin receptor agonists on leukocyte and haematopoietic stem and progenitor cells in the peripheral blood of patients with immune thrombocytopenic purpura. ANN HEMATOL. 2017: 1-12.

23. He S, Liu F, Lei X, et al. Protective Effects of Ferulic Acid against Heat Stress-Induced Intestinal Epithelial Barrier Dysfunction In Vitro and In Vivo. PLOS ONE. 2016; 11: e145236. 